\title{
Электронные формуляры для компьютерных тренажеров
}

\author{
А.В. Вус ${ }^{1,}$ преподаватель, 79218567422@yandex.ru \\ B.A. Ильин 1, д.в.н., профессор, старший научный сотрудник НИА, komandor.99@таil.ru
}

1 Военный институт дополнительного профессионального образования

ВУНЦ ВМФ "Военно-морская академия", г. Санкт-Петербург, 1951 12, Россия

\begin{abstract}
В статье предлагается использовать электронный формуляр для автоматизации основных процессов ведения формуляра на компьютерный тренажер при его обслуживании и для выработки предложений по его дальнейшему использованию на основе анализа собранных данных эксплуатации.

Электронный формуляр является аналогом стандартного формуляра. Предлагается общая структура электронного формуляра компьютерного тренажера, в БД которого будет храниться информация трех типов: постоянные данные, эксплуатационные данные и справочная информация. Предложен алгоритм работы электронного формуляра. Для сокращения ручных операций по сбору и обработке информации о состоянии тренажера и исключения влияния человеческого фактора на качество ведения технической документации в состав электронного формуляра включен модуль автоматического сбора и обработки данных. Этот модуль представляет собой систему клиент-сервер, устанавливаемую на все автоматизированные рабочие места компьютерного тренажера и позволяющую автоматизировать процессы контроля и анализа технического состояния компьютерного тренажера.

Впервые предложен алгоритм работы модуля автоматического сбора и обработки данных. Он включает в себя алгоритм работы клиента модуля, собирающего эксплуатационные данные, и сервера модуля, аккумулирующего и анализирующего полученные данные.

Применение предлагаемого модуля автоматического сбора и обработки информации позволит получать рекомендации по дальнейшему использованию компьютерного тренажера на основе собранной информации. Сформирован обобщенный алгоритм работы электронного формуляра с модулем автоматического сбора и обработки данных.

Предложенный алгоритм электронного формуляра дает возможность значительно снизить нагрузку на персонал, обслуживающий компьютерный тренажер, позволяя сконцентрировать внимание на подготовке тренажера к занятиям и их проведении.

Ключевые слова: компьютерный тренажер, специальное программное обеспечение, электронный формуляр, техническое обслуживание, качество технического обслуживания, алгоритм, автоматизация процессов эксплуатации, образовательное учреждение, образовательный прочесс, тренажерная подготовка.
\end{abstract}

Современный компьютерный тренажер представляет собой аппаратно-программный комплекс, который может включать от одного до нескольких десятков и даже сотен рабочих мест, объединенных в одно-, двух- или трехранговую вычислительную сеть [1]. В образовательном учреждении компьютерный тренажер может работать в различных конфигурациях рабочих мест с различной нагрузкой в разные периоды $[2,3]$. В таких условиях ведение обслуживающим персоналом обычных бумажных формуляров является довольно рутинной работой, занимающей значительное время, к тому же с возможными ошибками. Этих недостатков лишены электронные формуляры [4].

Общие требования к электронному формуляру определены ГОСТ Р 2.612-2011.

Для удобства работы пользователя с электронным формуляром в его структуре можно выделить два модуля: непосредственно БД и интерфейс работы с ней.

БД электронного формуляра хранит данные трех типов: справочная информация, постоянные данные и эксплуатационные данные $[5,6]$.

Использование электронного формуляра компьютерного тренажера имеет некоторые особенности, связанные со спецификой его построения и эксплуатации: большое число автоматизирован- ных рабочих мест (АРМ), изменение их конфигурации для проведения различных видов учебных занятий, особенности загружаемой информации на каждое учебное занятие и ряд других в зависимости от образовательных программ [7]. Предлагаемый вариант алгоритма работы пользователя компьютерного тренажера с интерфейсом электронного формуляра представлен на рисунке 1 .

Алгоритм работы с интерфейсом начинается с процедуры идентификации и аутентификации пользователя. Удостоверение всех эксплуатационных и ремонтных записей выполняют с помощью электронной цифровой подписи должностного лица, вносящего информацию в электронный формуляр, допускается вместо электронной цифровой подписи использовать информационно-удостоверяющий лист. После прохождения идентификации и аутентификации определяется дальнейший вид работ, производимых в ручном режиме.

Ручная запись новых эксплуатационных данных подразумевает ввод информации, которую нецелесообразно или невозможно собрать в автоматическом режиме. К ней относятся данные о консервации и расконсервации тренажера, движении тренажера в эксплуатации, изменениях в комплектации, выполненных работах и ремонтах, выявленных неисправностях. 


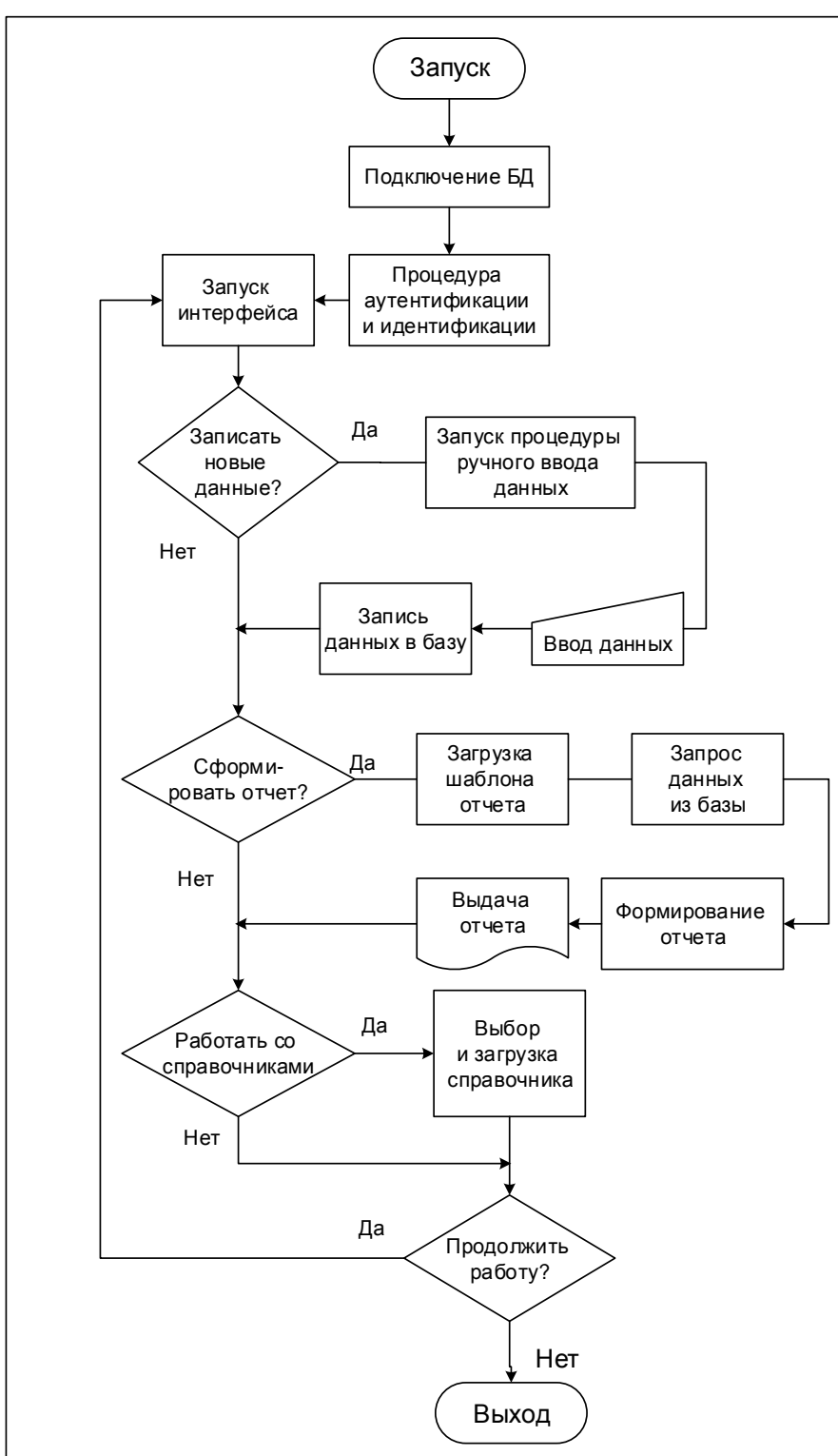

Рис. 1. Алгоритм работы с интерфейсом электронного формуляра

Fig. 1. Working with the electronic form interface

Формирование отчетов - это процесс получения информации из БД в формализованном виде для последующей обработки оператором или передачи в вышестоящий орган для обобщения и анализа. Для формирования отчета указывается вид или шаблон отчета, хранящийся в БД. Затем в соответствии с формой отчета загружаются необходимые данные из БД. В качестве отчетов могут выступать справки по применению тренажера по назначению, отчет о наработке тренажера по месяцам и нарастающим итогом, отчет об изменениях в комплектации тренажера с указанием причин и дат, отчет о техническом обслуживании и ремонтах. Одним из видов отчетов также могут быть данные, подготовленные для автоматизированного обмена технической информацией.
В режиме работы с интерфейсом также предусмотрена возможность работы со справочной информацией, заложенной в БД в виде справочников и интерактивных электронных технических руководств.

Очевидно, что использование электронных формуляров также предполагает довольно значительный объем работ по сбору и ручному заполнению электронных форм. Для автоматизации процесса сбора данных о работе тренажера при выполнении им функций по назначению в состав электронного формуляра предлагается ввести дополнительный модуль автоматического сбора и обработки данных.

Поскольку компьютерный тренажер строится на базе локальной вычислительной сети, модуль автоматического сбора и обработки данных представляет собой комплексную программу типа клиент-сервер $[6,8]$. Программа «клиент» инсталлируется вместе со специальным ПО тренажера на каждое АРМ, в том числе на рабочее место руководителя обучения, и предназначена для сбора информации и отправки ее на сервер. Сервер модуля автоматического сбора и обработки данных в составе электронного формуляра инсталлируется на рабочее место руководителя обучения или сервер (вычислительномоделирующий комплекс) тренажера и предназначен для сбора поступающей от клиентских программ информации и записи ее в БД электронного формуляра.

Обобщенный алгоритм работы клиента модуля автоматического сбора и обработки данных представлен на рисунке 2.

Клиент модуля автоматического сбора и обработки данных запускается вместе с включением AРМ тренажера. Для корректного определения любого события на рабочем месте важную роль играет синхронизация времени, в соответствии с этим первым действием клиента после включения является проверка текущего времени и его синхронизация с сервером автоматического сбора и обработки данных.

Далее клиент модуля проверяет корректность завершения предыдущей сессии тренажера по системному журналу операционной системы. После этого запускается цикл мониторинга тренажера.

Цикл мониторинга запускается с установленной периодичностью, например, раз в минуту. Интервал мониторинга можно изменять для различ- 


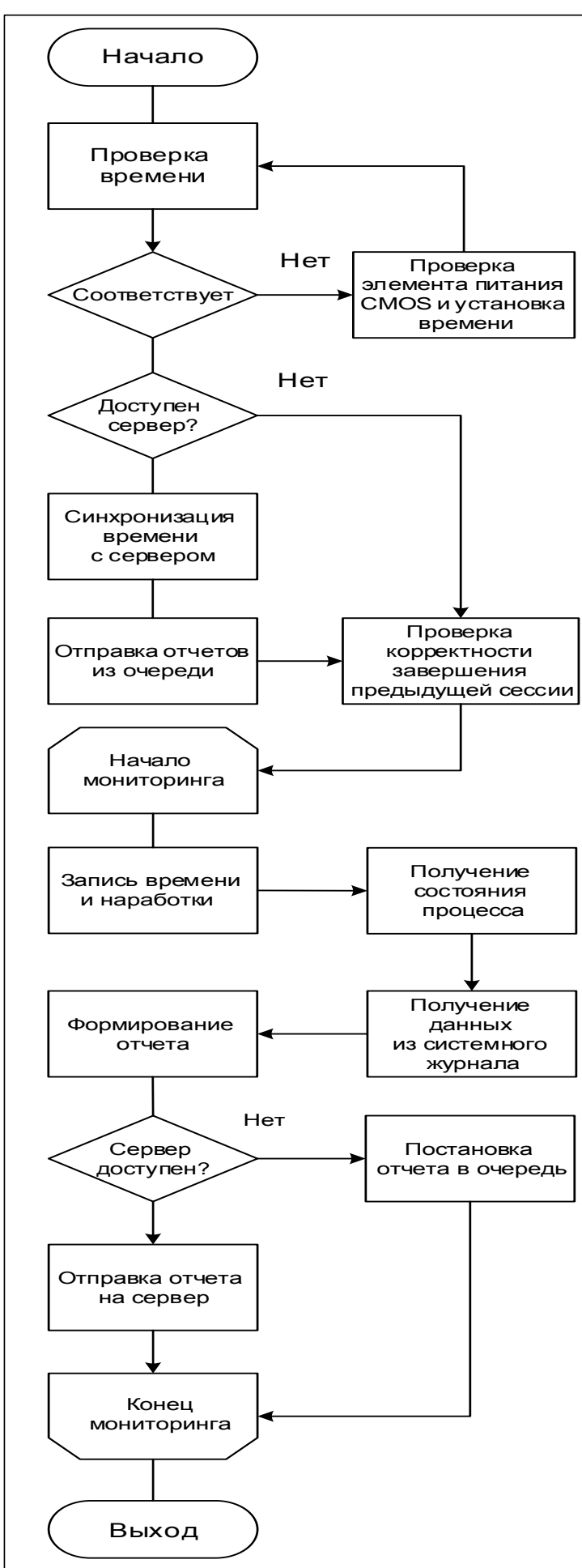

Рис. 2. Алгоритм работы клиента модуля АСОД

Fig. 2. An operation algorithm of the DPAS module client

ных режимов использования тренажера. При отладке и проверке работоспособности тренажера интервал запуска целесообразно сократить, а при проведении занятия, наоборот, увеличить для снижения нагрузки на вычислительные мощности рабочего места и пропускную способность локальной вычислительной сети.
Цикл мониторинга начинается с определения текущего времени и текущей наработки АРМ тренажера. Далее определяется состояние тренажера. Каждая программа, запущенная на АРM, в том числе специальное ПО тренажера, порождает несколько процессов в операционной системе, при этом необходимо отслеживать корректность их работы. Корректность работы тренажера определяется количеством сбоев процессов специального ПО тренажера на каждом АРМ.

Для отслеживания состояния общего ПО, влияющего на определение работоспособности тренажера, используется системный журнал операционной системы. Определяются критичные для функционирования тренажера события системного журнала, которые затем отслеживаются и передаются на сервер.

Для передачи собранных данных на сервер автоматического сбора и обработки данных используются формализованные отчеты следующего вида: $I D_{A R M} ; T ; T_{n} ; I D_{1 p r}=N_{p r} ; \ldots, I D_{n p r}=N_{n p r} ; I D_{s y s}=$ $=N_{\text {sys }} ; \ldots, I D_{n s y s}=N_{n s y s}$, где $I D_{A R M}-$ идентификатор AРМ в системе модуля автоматического сбора и обработки данных; $T$ - текущее время; $T_{n}$ - наработка АРМ тренажера; $I D_{l p r}$ - идентификатор 1-го процесса тренажера согласно таблице идентификаторов; $N_{l p r}-$ количество зависаний 1-го процесса; $I D_{n p r}$ - идентификатор $n$-го процесса тренажера согласно таблице идентификаторов; $N_{n p r}-$ количество зависаний $n$-го процесса; $I D_{1 s y s}-$ идентификатор 1-го системного события, влияющего на работу тренажера, согласно таблице идентификаторов; $N_{1 s y s}$ - количество появлений 1-го системного события; $I D_{\text {nsys }}$ - идентификатор $n$-го системного события, влияющего на работу тренажера, согласно таблице идентификаторов; $N_{\text {nsys }}-$ количество появлений $n$-го системного события.

В конце каждого цикла мониторинга сформированный отчет должен быть отправлен на сервер модуля автоматического сбора и обработки данных. Если сервер недоступен, отчет записывается в очередь на отправку и отправляется по мере доступа к серверу.

Обобщенный алгоритм работы сервера модуля автоматического сбора и обработки данных представлен на рисунке 3.

Данный сервер модуля запускается вместе с включением АРМ тренажера, на котором установлен электронный формуляр. Для корректного определения любого события тренажера важную роль играет синхронизация времени сервера модуля автоматического сбора и обработки данных со всеми клиентами модуля, установленными на тренажере. В соответствии с этим первыми действиями сервера модуля после включения являются проверка текущего времени и его синхронизация со всеми клиентами модуля автоматического сбора и обработки данных тренажера.

Далее происходят опрос АРМ, находящихся в сети, и выдача результатов. 


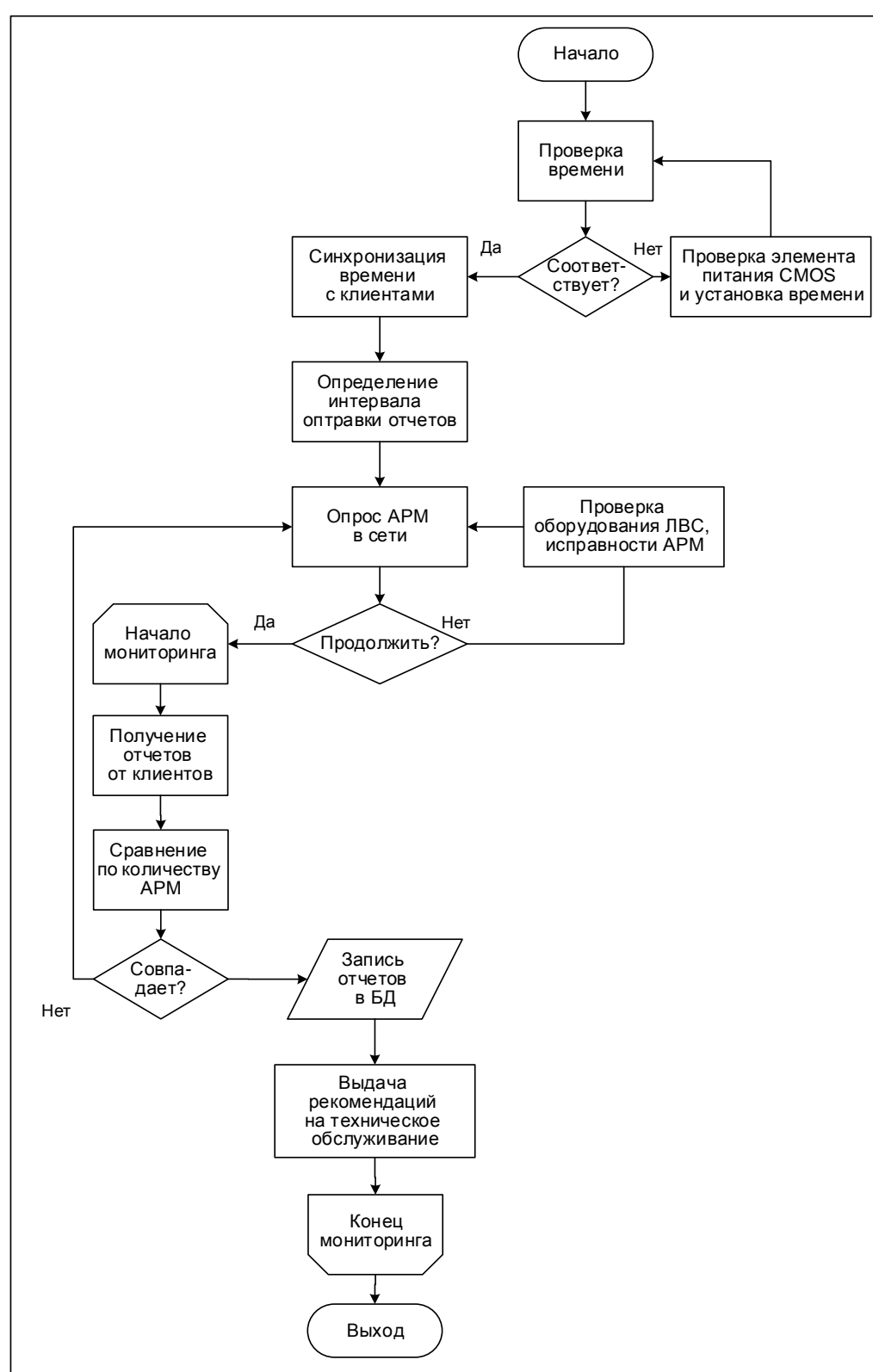

Рис. 3. Алгоритм работы сервера модуля АСОД

Fig. 3. An operation algorithm of the DPAS module server

Процесс мониторинга на сервере модуля автоматического сбора и обработки данных заключается в сборе отчетов от клиентов модуля и запись их в БД. Необходимо предусмотреть возможность пропуска отчета одним или несколькими АРМ. Для этого в процессе мониторинга необходимо сравнивать количество поступивших отчетов с количеством АРМ, запущенных в начале работы тренажера.

В процессе мониторинга в зависимости от наработки или срока службы одного АРМ или всего тренажера в целом могут быть выданы рекомендации по проведению планового технического обслуживания или планового ремонта [9].

Важным показателем при сборе информации о состоянии тренажера в определенный момент явля- ется конфигурация загруженного занятия. Определение конфигурации может происходить путем определения и записи работающих процессов на каждом АРМ либо ведением журнала загруженных конфигураций на рабочем месте руководителя обучения и сопоставлением записанной конфигурации со временем наступления события. Конфигурация тренажера может стать определяющим показателем при определении проблем в работе специального ПО

Обобщенный алгоритм работы электронного формуляра с модулем автоматического сбора и обработки данных представлен на рисунке 4. После запуска тренажера происходит подключение БД электронного формуляра. Далее запускается модуль автоматического сбора и обработки данных и работает в фоновом режиме вне зависимости от действий пользователя. Вместе с тем пользователь при необходимости запускает интерфейс электронного формуляра, проходит процедуру идентификации и аутентификации для работы. При работе с интерфейсом пользователь может вводить новые эксплуатационные данные, формировать отчеты для анализа эксплуатации и передачи данных.

Таким образом, применение электронного формуляра с модулем автоматического сбора и обработки данных позволит существенно автоматизировать процесс обслуживания компьютерного тренажера, что приведет к снижению вероятности появления ошибок при ведении формуляра, сконцентрировать внимание технического персонала на обеспечении проведения занятий на компьютерном тренажере, его техническом обслуживании и ремонте. Кроме того, появляется возможность достаточно точно получать статистический материал о состоянии тренажера, прогнозировать его работоспособность, вырабатывать предложения для совершенствования ПО, оптимизации номенклатуры и структуры аппаратных средств, что, очевидно, будет способствовать повышению качества подготовки военных специалистов с применением компьютерных тренажеров.

Предлагаемый модуль автоматического сбора и обработки данных в составе электронного формуляра с соответствующими модификациями может 


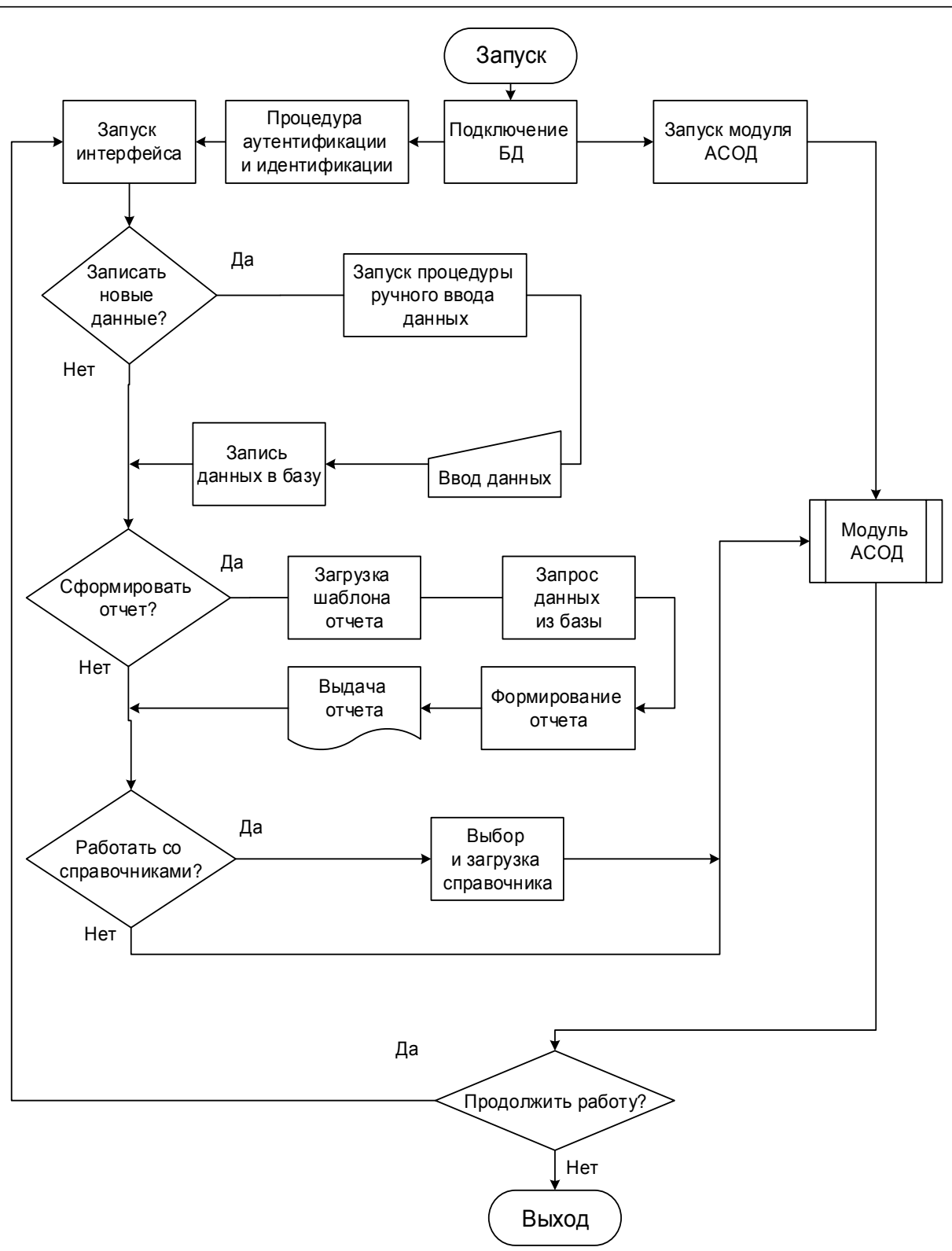

Рис. 4. Алгоритм работы электронного формуляра с модулем АСОД

Fig. 4. An algorithm of an electronic form working with the DPAS module

быть использован и в других сложных автоматизированных системах, построенных на базе локальных вычислительных сетей.

\section{Литература}

1. Алтунин В.К., Стручков А.М. Проектирование компьютерных систем обучения и интеллектуальной тренажерной подготовки специалистов Военно-морского флота. Тверь: Центрпрограммсистем, 2004. 204 с.

2. Базлов А.Ф., Рисунков В.Б., Соколов С.Н., Стручков А.М. Опыт разработки учебно-тренировочных средств для Военно-морского флота // Программные продукты и системы. 2016. № 1. C. 32-36. DOI: 10.15827/0236-235X.113.027-031.

3. Гончаров А.М., Крощук А.А., Ланчев В.М. Научно-технические проблемы создания и развития учебно-тренировочных средств обучения личного состава Войск воздушно-космической обороны // Военная мысль. 2018. № 1. С. 74-81.

4. Киричук И.А., Брусникин Е.В., Лепешинский И.Ю., Пе- пеляев А.В., Герасимов С.Д. Электронный формуляр // Навигатор в мире науки и образования. 2012. № 4-7. С. 622-623.

5. Дорошенко А.Б., Короленко В.В. Применение реляционных баз данных в системах информационной поддержки эксплуатации авиационной техники // Воздушно-космические силы. Теория и практика. 2017. № 1. С. 280-291.

6. Елизаров В.И., Галеев Э.Р., Мущинин А.В., Смолин Н.Г., Валеев И.М. Состав и структура распределенного компьютерного тренажера // Вестн. Казанского технологич. ун-та. 2013. № 20. C. 288-291.

7. Кеменов А.Ф., Кошелева Т.Н. Инструментальные средства предтренажерной и тренажерной подготовки операторов сложных технических систем // Программные продукты, системы и алгоритмы. 2014. № 2. URL: http://www.swsysweb.ru/tools-simulator-training-operators.html (дата обращения: 11.09.2018). DOI: 10.15827/2311-6749.11.130.

8. Долгова Е.В., Курушин Д.С., Федоров А.Б., Бикметов Р.Р. Организация сетевого взаимодействия элементов мобильного тренажерного комплекса // Инженерный вестн. Дона. 
2012. № 4. URL: http://www.ivdon.ru/ru/magazine/archive/n4p1y 2012/1266 (дата обращения: 11.09.2018).

9. Боев С.Ф., Игнатьев С.В., Тихонов В.Б., Беляков Р.А., Линкевичус А.П. Надежность и эксплуатация радиотехнических систем. М.: Научная книга, 2018. 370 с.

10. Вус А.В., Ильин В.А. Информационная поддержка процессов эксплуатации компьютерных тренажерных систем военного назначения // Проблемы развития и применения средств противовоздушной обороны на современном этапе. Средства противовоздушной обороны России и других стран мира, срав- нительный анализ: сб. докл. XVII Bсерос. науч.-практич. конф. Ярославль, 2016. С. 26-29.

11. Вус А.В. Модель сервисного обслуживания компьютерных тренажеров военного назначения // Программные продукты и системы. 2017. № 4. С. 785-789.

12. Андреев В.Ю., Ильин В.А., Базлов А.Ф., Шуванов А.Д Некоторые технологические аспекты создания учебно-тренировочных средств подготовки командиров и специалистов ВМФ // Программные продукты и системы. 2016. № 1. С. 32-36. DOI: 10.15827/0236-235X.113.032-036.

\section{Electronic forms for computer simulators}

A.V.Vus ${ }^{1}$, Teacher, 79218567422@yandex.ru

V.A. Ilin ${ }^{1}$, Dr.Sc. (Military Sciences), Professor, Senior Researcher of the Scientific Research Laboratory, komandor.99@mail.ru

${ }^{1}$ Military Institute of Supplementary Vocational Education VUNTs Navy "Naval Academy", St. Petersburg, 195112, Russian Federation

Abstract. The paper proposes to use an electronic form for automating the main processes of form transaction for a computer simulator during its maintenance and developing proposals for its further use based on the collected operation data analysis.

An electronic form is an electronic equivalent of a standard form. The paper presents a general structure of an electronic form for a computer simulator. It is proposed to store three types of information in an electronic form database. They are: permanent data, operational data and reference information. The paper also describes an operation algorithm for the electronic form. In order to reduce manual operations for collecting and processing data on a simulator state and to eliminate the the human factor affecting the quality of technical documentation, it is proposed to include an automatic data collection and processing module into the electronic form. This module is a client-server system installed on all computer simulator workstations. It allows automating the process of monitoring and analyzing a computer simulator technical state.

For the first time, the paper proposes an algorithm of an automatic data collection and processing module. It includes the algorithm of a module client collecting operational data and module servers accumulating and analyzing the obtained data.

The application of the proposed module for automatic data collection and processing will allow receiving recommendations on further use of a computer simulator based on the collected information. There is also a generalized algorithm for an electronic form operating with an automatic data collection and processing module.

The proposed algorithm of the electronic form will significantly reduce the load on computer simulator staff, therefore, it will allow focusing on preparing a simulator for classes and conducting classes.

Keywords: operation, computer simulator, special software, electronic form, maintenance, maintenance quality, algorithm, automation of operation processes, educational institution, educational process, simulator training.

\section{References}

1. Altunin V.K., Struchkov A.M. Designing Computer Training Systems and Intellectual Simulator Training for Navy Specialists. Tver, Centerprogramsystem Publ., 2004, 204 p.

2. Bazlov A.F., Risunkov V.B., Sokolov S.N., Struchkov A.M. An experience in development of educational training equipment for the Navy. Software \& Systems. 2016, no. 1, pp. $27-31$ (in Russ.). DOI: 10.15827/0236-235X.113.027-031.

3. Goncharov A.M., Kroshchuk A.A., Lanchev V.M. Scientific and technical problems of creation and development of training aids for training Aerospace Defense Forces personnel. Military Thought. 2018, no. 1, pp. 74-81 (in Russ.)

4. Kirichuk I.A., Brusnikin E.V., Lepeshinsky I.Yu., Pepelyaev A.V., Gerasimov S.D. Electronic form. The Navigator in the World of Science and Education. 2012, no. 4-7, pp. 622-623 (in Russ.).

5. Doroshenko A.B., Korolenko V.V. Relational databases in the systems of information support for the operation of aviation equipment. Aerospace Forces. Theory and Practice. 2017, no. 1, pp. 280-291 (in Russ.).

6. Elizarov V.I., Galeev E.R., Muschinin A.V., Smolin N.G., Valeev I.M. The composition and structure of a distributed computer simulator. Herald of Kazan Technological Univ. 2013, no. 20, pp. 288-291 (in Russ.).

7. Kemenov A.F., Kosheleva T.N. Tools for pre-training and simulator training of operators of complex technical systems. Software Products, Systems and Algorithms. 2014, no. 2. Available at: http://www.swsys-web.ru/tools-simulator-training-operators.html (accessed September 11, 2018). DOI: 10.15827/2311-6749.11.130.

8. Dolgova E.V., Kurushin D.S., Fedorov A.B., Bikmetov R.R. Mobile training complex network infrastructure. Engineering J. of Don. 2012, no. 4. URL: http://www.ivdon.ru/ru/magazine/archive/n4p1y2012/1266 (accessed September 11, 2018).

9. Boev S.F., Ignatiev S.V., Tikhonov V.B., Belyakov R.A., Linkevichus A.P. Reliability and Operation of Radio Engineering Systems. Moscow, Nauchnaya kniga Publ., 2018, 370 p.

10. Vus A.V., Ilyin V.A. Information support for the operation of military computer simulator systems. Problems of the Development and Application of Air Defense in Modern Times. Air Defence Facilities of Russia and Other Countries, Comparative Analysis: Proc. 17th All-Russian Sci. and Pract. Conf. Yaroslavl, 2016, pp. 26-29 (in Russ.).

11. Vus A.V. A service model for military computer simulators. Software \& Systems. 2017, no. 4, pp. 785-789 (in Russ.).

12. Andreev V.Yu., Ilin V.A., Bazlov A.F., Shuvanov A.D. Some technological aspects of creating educational training equipment for Navy commanding officers and specialists. Software \& Systems. 2016, no. 1, pp. 32-36 (in Russ.). DOI: 10.15827/0236235X.113.032-036. 